\title{
Conservation news
}

\section{INTRINSIC: Training materials for integrating rights and social issues in conservation}

Fauna \& Flora International, together with BirdLife International, the Tropical Biology Association and the Department of Geography, University of Cambridge, UK, have developed a flexible package of training materials to help build the capacity of conservationists to address the social and rights aspects of conservation. The project, known as INTRINSIC, was funded by the Cambridge Conservation Initiative Collaborative Fund. Materials were tested with students on the Cambridge University Masters in Conservation Leadership and with conservation practitioners in East Africa.

Biodiversity conservation is essentially a social process, involving as it does decisions about access to, and the use, values and protection of nature. As such, conservation inevitably entails both social costs and benefits, and the social context is likely to affect the efficiency and effectiveness of any conservation initiative. Unfortunately conservation practitioners often lack the knowledge and skills to address and integrate rights and other social issues into their work. The typical pathway to a professional conservation career involves the pursuit of educational opportunities and formal qualifications in natural sciences. However, the contemporary practice of conservation necessitates working with people, local communities and groups, and requires knowledge and skills about social systems that are often not gained through these traditional academic pathways. A number of authors have pointed out discrepancies between conservation course content and the skills needed, and have bemoaned the lack of training in the social dimensions of conservation (e.g. Saberwal \& Kothari, 1996, Conservation Biology, 10, 1328-1331; Jacobsen \& McDuff, 1998, Conservation Biology, 12, 263-267; Fisher et al., 2009, Oryx, 43, 361-363). The INTRINSIC training package aims to help address this capacity gap and improve conservation policy and practice by increasing environmental and social sustainability, thereby enabling positive, equitable outcomes for both nature and people.

The INTRINSIC materials, which can be found at http:// bit.ly/IntrinsicManual, comprise a trainers' guide and accompanying set of slide presentations designed to be customized for the particular context in which the training is to take place. It is expected that users will have some experience of working in conservation but in-depth knowledge of the specific social issues covered in the guide is not required. The developers envisage that delivery of the training can be a learning opportunity for trainers as well as participants. Subjects covered include community and social diversity, gender, conflict management, livelihoods and well-being. A range of governance topics are also covered, including rights-based approaches to conservation, and issues of equity, participation and power. The materials provided are designed for a 3-day course, or 4 days if a field trip is included. However, depending on the learning needs of participants, and the time available, each module can also be used independently or trainers can choose to deliver a subset of the modules. The trainers' notes for each module include session objectives, rationale (as background for the trainer), key learning points and step-by-step guidance for the delivery of the content, including plenary presentations. There is also a range of interactive activities and exercises involving case studies, role play, pair and small group discussions and feedback.

\section{Helen SCHNeIDer Fauna \& Flora International, Cambridge, UK E-mail helen.schneider@fauna-flora.org}

\section{Design and testing of a replicable, scalable capacity-building model for species conservation}

A major challenge for Venezuelan conservation scientists is to provide support to policy makers engaged in the conservation and sustainable use of the country's rich biological diversity, with comparably limited human and financial resources to do so. This challenge includes documenting the distribution and abundance of genes, species and ecosystems; integrating data from traditional knowledge and biological inventories; systematizing, analysing and socializing this information with the active involvement of key stakeholders; and facilitating open access through information and communication technologies.

A primary goal of the Biological Diversity Unit of the Venezuelan Institute for Scientific Investigation (IVIC) is to help by implementing a programme for building technical capacity in field sampling and data gathering techniques, and management of biological collections and information at the national scale, within the framework of the National Strategy for the Conservation of Biodiversity 2010-2020 and National Action Plan, with the vision of scaling it up regionally, as additional funds are secured.

Building on the facilities and infrastructure provided by IVIC, the Biological Diversity Unit has piloted a series of inter-institutional courses to develop the human resources required, focusing on skills that are relevant to providing inputs to Venezuela's reporting commitments to international agreements such as the Convention on Biological Diversity, CITES and the Sustainable Development Goals.

Courses on niche models and species distributions were offered during 2012-2015, georeferencing in 2014, and the 